\title{
SOME RESULTS CONCERNING THE EIGENVALUE PROBLEM FOR THE $p$-LAPLACIAN
}

\author{
Tilak Bhattacharya
}

\section{Introduction}

Let $\Omega$ be a bounded $C^{2}$ domain in $R^{n}, n \geq 2$. Consider the following problem

$$
\begin{aligned}
& L_{p} u+\lambda|u|^{p-2} u=0 \quad \text { in } \Omega \\
& u \in W_{0}^{1, p}(\Omega), u \not \equiv 0, \quad \lambda \in R \quad \text { and } 1<p<\infty,
\end{aligned}
$$

where $L_{p} u \equiv \operatorname{div}\left(|\nabla u|^{p-2} \nabla u\right)$ is the $p$-Laplacian. We say $u$ is a solution of (1.1) if there exists a $\lambda$ such that (1.1) holds in the sense of distributions, i.e.

$$
\int_{\Omega}|\nabla u|^{p-2} \nabla u \cdot \nabla \psi=\lambda \int_{\Omega}|u|^{p-2} u \psi \quad \forall \psi \in W_{0}^{1, p}(\Omega) .
$$

It is well known that there is a minimization problem related to (1.1), namely,

$$
\inf I(v), \quad v \in W_{0}^{1, p}(\Omega) \quad \text { and } J(v)=1,
$$

where $I(v)=(1 / p) \int_{\Omega}|\nabla v|^{p}$ and $J(v)=(1 / p) \int_{\Omega}|v|^{p}$. Then the following result holds [11].

Theorem 0. There exists a smallest $\lambda_{1}>0$ and an associated solution $u_{\lambda_{1}} \geq 0$ that solves (1.1). Furthermore, $\lambda_{1}$ is the infimum in (1.3).

We will refer to (1.1) as the eigenvalue problem for the $p$-Laplacian. The smallest eigenvalue $\lambda_{1}$ will be referred to as the first eigenvalue. Thelin in [11] shows that if $\Omega$ is a ball then $u_{\lambda_{1}}^{*}$, the spherically decreasing rearrangement of a solution $u_{\lambda_{1}}$, is also a solution. Furthermore, all radial solutions are unique up to scalar multiples. He then raises the question as to whether or not the first eigenfunction on the ball is radially symmetric. We showed in [2] that the answer is indeed yes, and the method was based on an idea due to Pólya and Szegö [8]. Let $\phi=u_{\lambda_{1}}$, where $u_{\lambda_{1}}$ is as in Theorem 0 , by the Hopf maximum principle $\phi>0$. Let $u$ be any other eigenfunction, define $f$ by $u=f \phi$. One then shows that $f$ is a constant. We have been able to extend this idea to prove a similar result on $C^{2}$ domains. The main difficulty here lies in showing that $f \in L^{\infty}(\Omega)$. This is achieved by the use of appropriate barriers. More precisely, we prove 
Theorem 1. Let $\Omega$ be a $C^{2}$ domain and $\lambda_{1}>0$, the first eigenvalue in (1.1), then $\lambda_{1}$ is simple.

Corollary 2.1. If $\Omega$ is a ball, then the first eigenfunction is radially symmetric.

Corollary 2.2. Let $\Omega$ be a $C^{2}$ domain and $\Omega^{\prime}$ a strict subdomain of $\Omega$, then $\lambda_{1}\left(\Omega^{\prime}\right)>\lambda_{1}(\Omega)$.

Corollary 2.3. Let $\Omega$ be as in Corollary 2.2, and $u$ an eigenfunction in (1.1) for some $\lambda_{0}>0$. If $u>0$ then $\lambda_{0}=\lambda_{1}$, i.e., eigenfunctions corresponding to higher eigenvalues must change sign in $\Omega$.

The second part of this paper is a study of the radial problem, when $\Omega$ is a ball of radius $R$. It is known that the eigenfunctions in (1.1) are $C_{\mathrm{loc}}^{1, \alpha}[3,12]$ and thus the radial eigenfunction $u(r)$ satisfies

$$
\begin{gathered}
|\dot{u}|^{p-2}\left\{(p-1) \ddot{u}+\frac{n-1}{r} \dot{u}\right\}+\lambda|u|^{p-2} u=0, \quad 0<r<R, \\
\dot{u}(0)=u(R)=0,
\end{gathered}
$$

where $\dot{u}$ and $\ddot{u}$ represent differentiations with respect to $r$. Our study primarily focuses on the distribution of higher eigenvalues in (1.4) [4]. In our work, instead of solving the problem on bounded domains, we consider the problem on all of $R^{n}$ with $\lambda=1$. We deduce that the solution, which we denote by $\phi(r)$, has countably many zeros and is globally unique. The zeros of $\phi$ can be related to the eigenvalues in (1.4) via a scaling argument, namely

$$
\lambda_{m+1}=\left(\frac{z_{m}}{R}\right)^{p}, \quad m=0,1,2, \ldots
$$

where $z_{m}$ is the $m$ th zero of $\phi$ and $\lambda_{m+1}$ is the $(m+1)$ th eigenvalue in (1.4). This shows that the radial problem has countably many eigenvalues and the uniqueness of $\phi$ proves that these are the only ones. Thus, we have

Theorem 2. For $1<p<\infty$, there is a unique $\phi \in C^{1}[0, \infty)$ that solves

$$
|\dot{\phi}|^{p-2}\left\{(p-1) \ddot{\phi}+\frac{n-1}{r} \dot{\phi}\right\}+|\phi|^{p-2} \phi=0, \quad r>0, \quad \phi(0)=1, \quad \dot{\phi}(0)=0
$$

and

(i) $\phi$ has countably many zeros $\left\{z_{m}\right\}_{m=1}^{\infty}$, ordered as $z_{0}<z_{1}<z_{2}<\cdots<z_{m}<$ $\cdots$, and $z_{m} \rightarrow \infty$ as $m \rightarrow \infty$,

(ii) $\lim _{r \rightarrow \infty}|\phi(r)|=0$, and

(iii) $\lim _{m \rightarrow \infty} z_{m+1}-z_{m}=T(p)$, 
where $T(p)=2(p-1)^{1 / p} \int_{0}^{1}\left(1-t^{p}\right)^{-1 / p} d t$. For $p \geq 2$,

$$
T(p)=\frac{2 \pi(p-1)^{1 / p}}{p \cos ((p-2) \pi / 2 p)} .
$$

For $p=2, \phi(r)$ is $r^{(2-n) / 2} J_{(n-2) / 2}(r)$, where $J_{(n-2) / 2}(r)$ is the Bessel function of order $(n-2) / 2$. It is interesting to note that for $p=2, T(2)=\pi$, a result well known about Bessel's functions.

While this work was being completed, the author was informed of several parallel works. Sakaguchi in [9] proves Theorem 1 for convex smooth domains; he also proves that if $u$ is the first eigenfunction then $\log |u|$ is a concave function. Anane also proves Theorem 1 for $C^{2, \alpha}$ domains [1]. The author thanks Professor Sakaguchi for pointing out the work of Anane. The author also thanks the referee for informing him of the work of Guedda-Veron [15] that contains results similar to Theorem 1. More recently, Azorero and Peral [5] have proven a general result regarding the asymptotic behaviour of the higher eigenvalues in (1.1). Finally, the author has learned that in a recent work Peter Lindqvist has a version of Theorem 1 valid in any domain.

\section{Proof of Theorem 1}

Let $\Omega$ be a bounded $C^{2}$ domain in $R^{n}, n \geq 2$, with $\partial \Omega$ connected. Then $\partial \Omega$ satisfies both an exterior and an interior sphere condition. Furthermore, one can find the largest ball that works for both cases. Let $R$ be the radius of such a ball. We introduce the following notation:

$$
\Omega_{h}=\{x \in \Omega: \operatorname{dist}(x, \partial \Omega) \geq h\} .
$$

Then $\operatorname{dist}\left(\partial \Omega_{h}, \partial \Omega\right)=h$.

Let $u$ be a solution of (1.1) in $\Omega$. It follows from [10; p. 264], for every $p$ such that $1<p \leq n$

$$
\|u\|_{\infty}<\infty
$$

and for $p>n, u \in C^{0, \alpha}(\bar{\Omega})$ with $\alpha=1-(n / p)$ [7; p. 163]. Again $u \in L^{\infty}(\Omega)$. Thus by the regularity results in $[3,12], u \in C_{\text {loc }}^{1, \beta}(\Omega)$ for some $\beta \in(0,1)$. By Hopf's maximum principle [13; p. 801], it follows that $u_{\lambda_{1}}$, in the statement of Theorem 0 , is strictly positive in $\Omega$.

Set $\phi=u_{\lambda_{1}}$, and let $u$ be any other eigenfunction corresponding to $\lambda=\lambda_{1}$ and satisfying (1.1). Define $f$ by $u=f \phi$, then $f \in C^{1}(\Omega)$ and $|f|$ is locally Lipschitz in $\Omega$.

Lemma 2.1. Let $\phi>0, u$ be eigenfunctions satisfying (1.1) with $\lambda=\lambda_{1}$. Let $f$ be defined by the equation $u=f \phi$, then $f \in L^{\infty}(\Omega)$. 
Proof. We divide the proof into three parts. Part (a) sets up an estimate for $u$ near $\partial \Omega$, using barrier functions. The construction of these functions is made possible by the exterior ball condition. Part (b) sets up a lower bound on the growth of $\phi$ near $\partial \Omega$. The proof follows the proof of Hopf's maximum principle, and uses the interior ball condition. Part (c) finishes the proof using the results of part (a) and part (b).

(a) We prove that

$$
|u(x)| \leq k_{0} \operatorname{dist}(x, \partial \Omega),
$$

where $x \in \Omega$ is such that $\operatorname{dist}(x, \partial \Omega) \leq R$, and $k_{0}$ depends on $n, p, R$ and $\|u\|_{\infty}$.

Let $x_{0} \in \partial \Omega$, then there is a $y_{0} \in \Omega^{c}$ such that the ball $B_{R}\left(y_{0}\right)$ lies outside $\Omega$ and $\partial B_{R}\left(y_{0}\right) \cap \partial \Omega=\left\{x_{0}\right\}$. Define

$$
w(x)=A\left(\frac{1}{R^{\sigma}}-\frac{1}{\left|x-y_{0}\right|^{\sigma}}\right)
$$

where $\sigma=(n-1) /(p-1)$, (we may choose $\sigma \geq(n-1) /(p-1)), x \in \Omega$ and $A$ a positive constant to be determined later. Then,

$$
L_{p} w=-\frac{(p-1)(\sigma A)^{p-1}}{r^{\sigma(p-1)+p}}
$$

where $r=\left|x-y_{0}\right|$. Let $S_{R}=B_{2 R}\left(y_{0}\right) \cap \Omega$, choose $A$ such that,

$$
\lambda_{1}\|u\|_{\infty}^{p-1} \leq \frac{(p-1)(\sigma A)^{p-1}}{(2 R)^{\sigma(p-1)+p}}, \quad \text { and } \quad\|u\|_{\infty} \leq A\left(\frac{1}{R^{\sigma}}-\frac{1}{(2 R)^{\sigma}}\right) .
$$

Then $w(x) \geq u(x)$ on $\partial S_{R}$ and $L_{p} w \leq L_{p} u$ in $S_{R}$. By the weak comparison principle [12], it follows that $w(x) \geq u(x)$ in $S_{R}$. Replacing $w$ by $-w$, we get that $|u(x)| \leq w(x)$. Hence,

$$
|u(x)| \leq A\left(\frac{1}{R^{\sigma}}-\frac{1}{\left|x-y_{0}\right|^{\sigma}}\right) \quad \forall x \in S_{R} .
$$

Set $r=\left|x-y_{0}\right|$, then by an application of the mean value theorem,

$$
\frac{1}{R^{\sigma}}-\frac{1}{r^{\sigma}} \leq \frac{\sigma(r-R)}{R^{\sigma+1}} \quad \text { in } \quad R<r<2 R .
$$

Thus for some $k_{0}>0$,

$$
|u(x)| \leq k_{0}\left(\left|x-y_{0}\right|-R\right) .
$$


Let $x \in \Omega$ be such that $\operatorname{dist}(x, \partial \Omega) \leq R$; then there is a $x_{0} \in \partial \Omega$ such that $\operatorname{dist}\left(x, x_{0}\right)=\operatorname{dist}(x, \partial \Omega)$. There is a corresponding $y_{0} \in \Omega^{c}$ and a ball $B_{R}\left(y_{0}\right)$ that satisfies the exterior ball condition at $x_{0}$. Then it follows that $\operatorname{dist}(x, \partial \Omega)=$ $\left|x-y_{0}\right|-R$, and hence

$$
|u(x)| \leq k_{0} \operatorname{dist}(x, \partial \Omega)
$$

(b) We now prove a lower bound for the growth of $\phi$ near $\partial \Omega$. We show that

$$
\phi(x) \geq k_{1} \operatorname{dist}(x, \partial \Omega),
$$

where $x \in \Omega$ and $\operatorname{dist}(x, \partial \Omega) \leq R / 2, k_{1}$ depends only on $n, p, R$ and $\phi$.

We start by presenting the proof of Hopf's maximum principle. From (2.1), it is clear that every eigenfunction is continuous up to the boundary. Thus $\phi$ is zero on $\partial \Omega$ in the classical sense. Let $x_{0} \in \partial \Omega$, and $B_{R}\left(z_{0}\right) \subset \Omega$ be such that $\partial B_{R}\left(z_{0}\right) \cap \partial \Omega=\left\{x_{0}\right\}$. Let $S=B_{R}\left(z_{0}\right) \backslash B_{R / 2}\left(z_{0}\right)$; take

$$
v_{z_{0}}(x)=e^{-\alpha\left|x-z_{0}\right|^{2}}-e^{-\alpha R^{2}}, \quad \forall x \in S .
$$

Thus for every $x \in S$,

$$
L_{p} v_{z_{0}}(x) \geq C e^{-\alpha(p-1) R^{2}}\left\{(p-1) \alpha^{2} R^{2}-2 \alpha(p+n-2)\right\},
$$

where

$$
C= \begin{cases}(\alpha R)^{p-2}, & \text { if } 2 \leq p<\infty \\ (2 \alpha R)^{p-2}, & \text { if } 1<p<2 .\end{cases}
$$

Choosing $\alpha$ large enough, it follows that $L_{p} v_{z_{0}} \geq 0 \geq L_{p} \phi$, in $S$. Since $\phi \in C^{1}(\Omega)$ and $\phi>0$, it follows that

$$
\lim _{\Omega_{R / 2}} \phi>0 .
$$

Thus, there is an $\epsilon>0$ such that $\epsilon v_{z_{0}}<\phi$ on $\partial B_{R / 2}\left(z_{0}\right)$, for all $z_{0} \in \partial \Omega_{R}$. Note that $v_{z_{0}}$ vanishes on $\partial B_{R}\left(z_{0}\right)$. Therefore, by the weak comparison principle, $\phi(x) \geq \epsilon v_{z_{0}}(x)$ in $S$. Again, by an application of the mean value theorem,

$$
\phi(x) \geq k_{1}\left(R-\left|x-z_{0}\right|\right) \quad \forall x \in S .
$$

Let $x \in \Omega \backslash \Omega_{R / 2}$, then there is an $x_{0} \in \partial \Omega$ such that $\operatorname{dist}\left(x, x_{0}\right)=\operatorname{dist}(x, \partial \Omega)$. There is a $z_{0}$ such that $x=t x_{0}+(1-t) z_{0}$ for some $t \in[0,1]$, and the ball $B_{R}\left(z_{0}\right)$ lies in $\Omega$ and $\partial B_{R}\left(z_{0}\right) \cap \partial \Omega=\left\{x_{0}\right\}$. Thus,

$$
\phi(x) \geq k_{1} \operatorname{dist}(x, \partial \Omega) .
$$

(c) To finish the proof, we note that in $\Omega_{R / 2}$,

$$
|f|=\frac{|u|}{\phi} \leq \frac{\|u\|_{\infty}}{\inf _{\Omega_{R / 2}} \phi}<\infty .
$$

From (2.1) and (2.3), it follows that in $\Omega \backslash \Omega_{R / 2}$,

$$
|f|=\frac{|u|}{\phi} \leq \frac{k_{0} \operatorname{dist}(x, \partial \Omega)}{k_{1} \operatorname{dist}(x, \partial \Omega)}<\infty .
$$

Hence $f \in L^{\infty}(\Omega)$. 
Remark 2.1. Estimates (2.1) and (2.3) hold for equations $L_{p} u+F(x, u)=0$ in $\Omega$ with $u \in W_{0}^{1, p}(\Omega), F \in L^{\infty}$ and $u>0$.

Lemma 2.2. We have that $|f|^{p} \phi \in W_{0}^{1, p}(\Omega)$.

Proof. We note that $|f|$ is Lipschitz continuous in $\Omega$. Furthermore, $|\nabla f|=$ $|\nabla| f||$ a.e. For $n=1,2,3, \ldots$, let $h_{n}=h / n$ and $\bar{h}_{n}=h_{n / 2}$, and $0 \leq \psi_{n} \leq 1$ be a function in $C_{0}^{1}(\Omega)$ such that

$$
\psi_{n}(x)=\left\{\begin{array}{ll}
1 & \text { in } \Omega_{h_{n}} \\
0 & \text { in } \Omega \backslash \Omega_{\bar{h}_{n}}
\end{array},\right.
$$

and $\left|\nabla \psi_{n}\right| \leq C n / h$, where $C$ is a universal constant and in general would depend on $\Omega$. From (2.1), $\phi(x) \leq K \operatorname{dist}(x, \partial \Omega)$ implying then $\phi(x) \leq K h / n$ in $\Omega \backslash \Omega_{h n}$. The rest of the proof is now the same as in Lemma 3 in [2].

Lemma 2.3. Let $f$, $\phi$ be $C^{1}$ functions, $1<p<\infty$, then

$$
|\nabla f \phi|^{p} \geq|\nabla \phi|^{p-2} \nabla \phi \cdot \nabla\left(f^{p} \phi\right)+K \phi^{p}|\nabla f|^{p},
$$

where $0 \leq K \leq 1$ and $K=0$ if and only if $\phi \nabla f=0$.

Proof. See Proposition 2 and Theorem 1 in [3].

Proof of Theorem 1. Let $\phi>0, u$ be eigenfunctions satisfying (1.1) with $\lambda=\lambda_{1}$. Let $f$ be defined by $u=f \phi$. The proof that $f$ is a constant and thus $\lambda_{1}$ is simple is exactly the same as the proof of Theorem 1 in [2]. It is clear that $u$ does not change sign in $\Omega$.

Proof of Corollary 2.1. Immediate.

Proof of Corollary 2.2. It is clear that $\lambda_{1}\left(\Omega^{\prime}\right) \geq \lambda_{1}(\Omega)$. Suppose equality holds. Let $u \in W_{0}^{1, p}\left(\Omega^{\prime}\right)$ be the nonnegative minimizer of (1.3) with $\Omega$ replaced by $\Omega^{\prime}$. Extend $u$ by zero to rest of $\Omega$. This modified $u$ is in $W_{0}^{1, p}(\Omega)$ and is a minimizer of (1.3) in $\Omega$. Clearly, $u \geq 0$ in $\Omega$ and by the results in [11], $u$ solves (1.1) with $\lambda=\lambda_{1}$ in $\Omega$. By Theorem $1, u>0$ in $\Omega$, and hence in $\Omega \backslash \Omega^{\prime}$, a contradiction.

Proof of Corollary 2.3. It is clear that $\lambda_{0} \geq \lambda_{1}$. Let $\phi>0$ be the first eigenfunction in (1.1). Define $f$ by $\phi=f u$. Then from Lemma 2.2, it follows that $f^{p} u$ is a legitimate test function. Proceeding as in Theorem 1 in [2],

$$
\int_{\Omega}|\nabla \phi|^{p}=\lambda_{1} \int_{\Omega} \phi^{p}
$$

and

$$
\int_{\Omega}|\nabla u|^{p-2} \nabla u \cdot \nabla\left(f^{p} \phi\right)=\lambda_{0} \int_{\Omega}(f u)^{p}=\lambda_{0} \int_{\Omega} \phi^{p}>0 .
$$


Comparing,

$$
\int_{\Omega}|\nabla \phi|^{p}=\frac{\lambda_{1}}{\lambda_{0}} \int|\nabla u|^{p-2} \nabla u \cdot \nabla\left(f^{p} u\right)
$$

Using Lemma 2.3,

$$
\int_{\Omega} K u^{p}|\nabla f|^{p} \leq 0
$$

Thus, $f$ is a constant and $\lambda_{0}=\lambda_{1}$. $\square$

\section{Proof of Theorem 2}

We will obtain the proof of Theorem 2 through several lemmas. Let $\phi$ satisfy

$$
\begin{gathered}
\frac{d}{d r}\left(r^{n-1}|\dot{\phi}|^{p-2} \dot{\phi}\right)+r^{n-1}|\phi|^{p-2} \phi=0, \quad 0<r<\infty, \\
\phi(0)=1 \quad \text { and } \quad \dot{\phi}(0)=0 \quad \text { and } 1<p<\infty,
\end{gathered}
$$

where $\dot{\phi}$ represents differentiation with respect to $r$. The choice $\phi(0)=1$ is arbitrary. The function $\phi$ defined through the following integral equations satisfies (3.1);

$$
\dot{\phi}(r)=g^{-1}\left(-\left\{\frac{1}{r^{n-1}} \int_{0}^{r} t^{n-1}|\phi(t)|^{p-2} \phi(t) d t\right\}\right)
$$

and

$$
\phi(r)=1+\int_{0}^{r} g^{-1}\left(-\left\{\frac{1}{t^{n-1}} \int_{0}^{t} s^{n-1}|\phi(s)|^{p-2} \phi(s) d s\right\}\right) d t
$$

where $g(\tau)=|\tau|^{p-2} \tau,-\infty<\tau<\infty$ and $g^{-1}(t)=|t|^{q-2} t$ with $(1 / p)+(1 / q)=$ 1. We note that the first zero of $\phi(r)$ as defined in (3.3), is the radius of the ball for which $\lambda=1$ is the first eigenvalue. For $p=2$, the function $\phi(r)$ is $r^{(2-n) / 2} J_{(n-2) / 2}$, where $J_{(n-2) / 2}$ is the Bessel function of order $(n-2) / 2$.

Lemma 3.1. The function $\phi(r)$, as defined in (3.3), has countably many zeros in $r>0$.

Proof. We change the problem in (3.1) in order to attain more generality. Let us specify the conditions in (3.1) at an arbitrary point $r=a$, with $a \geq 0$, i.e. we take $\phi(a)=1$ and $\dot{\phi}(a)=0$. The corresponding integral equations for $\phi$ become

$$
\dot{\phi}(r)=-\left\{\frac{1}{r^{n-1}} \int_{a}^{r} t^{n-1}|\phi(t)|^{p-2} \phi(t) d t\right\}^{1 /(p-1)},
$$


and

$$
\phi(r)=1-\int_{a}^{r}\left\{\frac{1}{t^{n-1}} \int_{a}^{t} s^{n-1}|\phi(s)|^{p-2} \phi(s) d s\right\}^{1 /(p-1)} d t,
$$

in $r>a$. We show that $\phi(r)$ as defined in (3.5) changes sign. Near $r=a, \phi$ is positive and $\dot{\phi}$ is negative. It follows that $\phi$ is decreasing and (3.4) may be rewritten as

$$
\begin{aligned}
|\dot{\phi}(r)|^{p-1} & =\frac{1}{r^{n-1}}\left\{\int_{a}^{b} t^{n-1}|\phi(t)|^{p-2} \phi(t) d t+\int_{b}^{r} t^{n-1}|\phi(t)|^{p-2} \phi(t) d t\right\} \\
& \geq \frac{1}{r^{n-1}}\left\{A+(\phi(r))^{p-1}\left(\frac{r^{n}-b^{n}}{n}\right)\right\},
\end{aligned}
$$

where $b>a$ is close to $a$, and $A=\int_{a}^{b} t^{n-1}|\phi(t)|^{p-2} \phi(t) d t$. Using the inequality $(x+y)^{1 /(p-1)} \geq C\left(x^{1 /(p-1)}+y^{1 /(p-1)}\right), x>0, y>0$ and $C$ an appropriate constant depending on $p$, we have

$$
|\dot{\phi}(r)| \geq C r^{(1-n) /(p-1)}\left\{A^{1 /(p-1)}+\phi(r)\left(\frac{r^{n}-b^{n}}{n}\right)^{1 /(p-1)}\right\} .
$$

Let $\bar{r}>b$ be such that $\left(r^{n}-b^{n}\right) / n \geq r^{n} / 2 n$, for $r>\bar{r}$. If $\phi(r)$ is zero for some $r \leq \bar{r}$, we are done. Otherwise continue $\phi$ past $r=\bar{r}$. With new constants $B$ and $C$, the above inequality for $\dot{\phi}$ becomes

$$
|\dot{\phi}(r)| \geq B r^{(1-n) /(p-1)}+C r^{1 /(p-1)} \phi(r), \quad \text { in } r>\bar{r} .
$$

Noting that $\dot{\phi} \leq 0$, an integration yields with new constants $D$ and $E$,

$$
\phi(r) \leq e^{-D r^{p /(p-1)}}\left\{E-\int_{\bar{r}}^{r} \frac{e^{D t^{p /(p-1)}}}{t^{(n-1) /(p-1)}} d t\right\} .
$$

Since the integral on the right side of the inequality is divergent, $\phi(r)$ changes sign at some $r$ in $(a, \infty)$. For $a=0$, call this point $z_{0}$. Thus $z_{0}$ is the first zero of $\phi(r)$ that solves (3.3). From (3.2), it is clear that $\dot{\phi}\left(z_{0}\right)<0$. Continue $\phi$ past $r=z_{0}$, using (3.3). In order to prove the next statement we may take without any loss of generality, $z_{0}=1$ and $\dot{\phi}\left(z_{0}\right)=-\delta$, where $\delta$ is any positive number. We now show that there is a $r_{1} \in(1, \infty)$ such that $\dot{\phi}(r) \rightarrow 0$ as $r \rightarrow r_{1}$.

It is clear that near $r=1, \dot{\phi}$ is negative, thus $\phi$ is decreasing and is negative. In a small righthand neighborhood of $r=1, \dot{\phi}$ satisfies

$$
\begin{aligned}
|\dot{\phi}(r)|^{p-1} & =\frac{1}{r^{n-1}}\left\{\delta^{p-1}+\int_{1}^{r} t^{n-1}|\phi(t)|^{p-2} \phi(t) d t\right\} \\
& \leq \frac{1}{r^{n-1}}\left\{\delta^{p-1}+\int_{1+\epsilon}^{r} t^{n-1}|\phi(t)|^{p-2} \phi(t) d t\right\},
\end{aligned}
$$


where $\epsilon>0$, is a small positive number. Noting that $\phi(1+\epsilon)<0$, we obtain

$$
|\dot{\phi}(r)|^{p-1} \leq \frac{1}{r^{n-1}}\left\{\delta^{p-1}-|\phi(1+\epsilon)|^{p-1}\left(\frac{r^{n}-(1+\epsilon)^{n}}{n}\right)\right\} .
$$

Thus there is a $r_{1} \in(1, \infty)$ such that $\dot{\phi}(r) \rightarrow 0$ as $r \rightarrow r_{1}$. Again continue $\phi$ past $r_{1}$ using (3.3). By repeating the foregoing arguments, it can be shown that $\phi$ has countably many zeros, $z_{0}<z_{1}<z_{2}<\cdots<z_{m}<\cdots$. Furthermore, $\phi$ attains its relative extrema where $\dot{\phi}$ vanishes. Label these as $h_{0}<h_{1}<h_{2}<\cdots<h_{m}<\ldots$, where $h_{0}=0$, and $h_{m}<z_{m}<h_{m+1}$. 口

To prove that the zeros march to infinity, we need the following lemma.

Lemma 3.2. The distance between two successive zeros is bounded uniformly from below.

Proof. For a fixed $m \geq 0$, consider the interval $\left[z_{m}, z_{m+1}\right]$. Without any loss of generality, we may take $\phi$ to be positive in this interval. The function $\phi$ is increasing in $\left[z_{m}, h_{m+1}\right]$ and decreasing in $\left[h_{m+1}, z_{m+1}\right]$. We show that $z_{m+1}-h_{m+1}$ is bounded from below, the proof for $h_{m+1}-z_{m}$ follows in a similar fashion. Let $\phi\left(h_{m+1}\right)=M, \ell$ any number in $(0,1]$; noting that $\dot{\phi}\left(h_{m+1}\right)=0$ and $\dot{\phi}(r) \leq 0$ in $\left[h_{m+1}, z_{m+1}\right]$, we have

$$
\phi(r)=M-\int_{h_{m+1}}^{r}\left\{\frac{1}{t^{n-1}} \int_{h_{m+1}}^{t} s^{n-1}|\phi(s)|^{p-2} \phi(s) d s\right\}^{1 /(p-1)} d t, \quad \text { in } r>h_{m+1} .
$$

Let $b_{\ell m+1} \in\left[h_{m+1}, z_{m+1}\right]$ be such that $\phi\left(b_{\ell m+1}\right)=\ell M$. Since $\phi$ is decreasing, $\ell M \leq \phi(r) \leq M$ in $\left[h_{m+1}, b_{\ell m+1}\right]$, thus

$$
\ell M \geq M-M \int_{h_{m+1}}^{b_{\ell m+1}}\left\{\frac{t^{n}-h_{m+1}^{n}}{n t^{n-1}}\right\}^{1 /(p-1)} d t .
$$

Using the inequality, $t^{n}-h_{m+1}^{n} \leq n t^{n-1}\left(t-h_{m+1}\right)$, and integrating once more, we have

$$
(1-\ell) \frac{p}{p-1} \leq\left(b_{\ell m+1}-h_{m+1}\right)^{p /(p-1)} .
$$

Thus,

$$
b_{\ell m+1}-h_{m+1} \geq I(\ell, p),
$$

where $I(\ell, p)$ is an appropriate constant depending on $\ell$ and $p$, and independent of $M$. व

Proof of part (i) of Theorem 2. From Lemmas 3.1 and 3.2, it follows that $z_{m} \rightarrow \infty$ as $m \rightarrow \infty$. ㅁ

We now prove results needed for discussing the asymptotics of $\phi$. 
Lemma 3.3. The distance between two successive zeros is bounded from above.

Proof. For a fixed $m$, consider the interval $\left[z_{m}, z_{m+1}\right]$. We will assume that $\phi$ is positive in this interval. As before, $\phi$ increases in $\left[z_{m}, h_{m+1}\right]$ and decreases in $\left[h_{m+1}, z_{m+1}\right]$. In part (a) we prove the assertion for the subinterval $\left[z_{m}, h_{m+1}\right]$, and in part (b) we treat the subinterval $\left[h_{m+1}, z_{m+1}\right]$. The proof of the latter is more involved and we need to treat the cases $1<p<n, p=n$ and $p>n$, separately. Let $\phi\left(h_{m+1}\right)=M>0$.

(a) Consider $\left[z_{m}, h_{m+1}\right]$, noting that $\phi\left(z_{m}\right)=\dot{\phi}\left(h_{m+1}\right)=0, \phi>0$ and $\dot{\phi}>0$ in this interval, it follows

$$
(\dot{\phi}(r))^{p-1}=\frac{1}{r^{n-1}}\left\{\left(z_{m}\right)^{n-1}\left(\dot{\phi}\left(z_{m}\right)\right)^{p-1}-\int_{z_{m}}^{r} t^{n-1}(\phi(t))^{p-1} d t\right\},
$$

for $z_{m} \leq r \leq h_{m+1}$. Thus $\dot{\phi}$ is decreasing and $\phi$ is concave in this subinterval. For the proof, we use the following form for $\dot{\phi}$.

$$
\dot{\phi}(r)=\left\{\frac{1}{r^{n-1}} \int_{r}^{h_{m+1}} t^{n-1}(\phi(t))^{p-1} d t\right\}^{1 /(p-1)} .
$$

Integrating (3.7) once from $z_{m}$ to $h_{m+1}$, and noting that $t \geq r$, we find

$$
\phi\left(h_{m+1}\right) \geq \int_{z_{m}}^{h_{m+1}}\left\{\int_{r}^{h_{m+1}}(\phi(t))^{p-1} d t\right\}^{1 /(p-1)} d r .
$$

Setting $T=h_{m+1}-z_{m}$ and using that $\phi(r) \geq\left(M\left(r-z_{m}\right)\right) / T$ (this follows from the concavity), the above integral inequality for $\phi$ yields

$$
\int_{z_{m}}^{h_{m+1}} \frac{1}{T}\left\{\int_{r}^{h_{m+1}}\left(t-z_{m}\right)^{p-1} d t\right\}^{1 /(p-1)} d r \leq 1 .
$$

After a few simplifications,

$$
\left(\frac{T^{p}}{p}\right)^{1 /(p-1)} \int_{0}^{1}\left(1-\tau^{p}\right)^{1 /(p-1)} d \tau \leq 1
$$

Thus,

$$
T \leq c(p),
$$


where $c(p)$ is an appropriate constant depending only on $p$. Thus $h_{m+1}-z_{m}$ is bounded from above uniformly.

(b) Now consider the interval $\left[h_{m+1}, z_{m+1}\right]$. We note that $\phi\left(z_{m+1}\right)=\dot{\phi}\left(h_{m+1}\right)$ $=0, \phi>0$ and $\dot{\phi}<0$ in this interval. By differentiating (3.3) twice, it can be shown that $\phi$ has a point of inflection. For $1<p<\infty$, let

$$
t= \begin{cases}r^{(p-n) /(p-1)}, & p \neq n \\ \ell n r, & p=n .\end{cases}
$$

Set $w(t)=\phi(r)$ for $r>0$. The differential equation in (3.1) is thus transformed to

$$
(p-1)\left|\frac{p-n}{p-1}\right|^{p}|\dot{w}|^{p-2} \ddot{w}+t^{(n-1) p /(p-n)}|w|^{p-2} w=0, \quad p \neq n
$$

and

$$
(n-1)|\dot{w}|^{n-2} \ddot{w}+e^{n t}|w|^{n-2} w=0, \quad p=n,
$$

where now the differentiations are with respect to $t$. It is clear that $w$ is concave whenever $w>0$. We now consider the three cases $1<p<n, p>n$ and $p=n$, separately.

Case 1. Consider $1<p<n$. Equation (3.9) holds in the interval $\left[T_{1}, T_{2}\right]$, where $T_{1}=\left(z_{m+1}\right)^{(p-n) /(p-1)}$ and $T_{2}=\left(h_{m+1}\right)^{(p-n) /(p-1)}$. Note that $\dot{w}\left(T_{2}\right)=$ $\dot{\phi}\left(h_{m+1}\right)=0, \operatorname{sign}(\dot{w})=-\operatorname{sign}(\dot{\phi})$ and $w$ is increasing. Integrating (3.9) twice, we get

$$
w(t)=w\left(T_{2}\right)-\frac{1}{A} \int_{t}^{T_{2}}\left\{\int_{x}^{T_{2}} s^{p(n-1) /(p-n)}|w(s)|^{p-2} w(s) d s\right\}^{1 /(p-1)} d x
$$

where $A=|(p-n) /(p-1)|^{p /(p-1)}$. Let $0<\delta \leq 1$, and $T_{\delta} \in\left[T_{1}, T_{2}\right]$ be such that $w\left(T_{\delta}\right)=(1-\delta) M$, where $w\left(T_{2}\right)=\phi\left(h_{m+1}\right)=M$. Taking $t=T_{\delta}$ in (3.11) and simplifying, we obtain

$$
\int_{T_{6}}^{T_{2}}\left\{\int_{x}^{T_{2}} s^{p(n-1) /(p-n)}|w(s)|^{p-2} w(s) d s\right\}^{1 /(p-1)} d x=A \delta M .
$$

By concavity, $w(s) \geq M\left(1+\left(s-T_{2}\right) \delta /\left(\bar{T}_{\delta}\right)\right)$ where $\bar{T}_{\delta}=T_{2}-T_{\delta}$; and thus from the aforementioned integral equality we get

$$
\int_{T_{\delta}}^{T_{2}}\left\{\int_{x}^{T_{2}} s^{(n-1) p /(p-n)}\left(1+\frac{s-T_{2}}{\bar{T}_{\delta}} \delta\right)^{p-1} d s\right\}^{1 /(p-1)} d x \leq A \delta .
$$


Since $s \leq T_{2}$ and $p<n, s^{(n-1) p /(p-n)} \geq T_{2}^{(n-1) p /(p-n)}$. Thus the above inequality after an integration yields,

$$
\left(T_{2}\right)^{(n-1) p /(p-n)(p-1)}\left(\frac{\bar{T}_{\delta}}{p \delta}\right)^{1 /(p-1)} \int_{T_{\delta}}^{T_{2}}\left[1-\left\{1+\frac{x-T_{2}}{\bar{T}_{\delta}}\right\}^{p}\right]^{1 /(p-1)} d x \leq A \delta .
$$

Setting $\tau=1+\left(x-T_{2}\right) \delta /\left(\bar{T}_{\delta}\right)$, we obtain

$$
\left(\frac{\bar{T}_{\delta} T_{2}^{(n-1) /(p-1)}}{\delta}\right)^{p /(p-1)} \int_{1-\delta}^{1}\left(1-\tau^{p}\right)^{1 /(p-1)} d \tau \leq A p^{1 /(p-1)} \delta .
$$

For $\delta \geq \frac{1}{2}$

$$
\int_{1-\delta}^{1}\left(1-\tau^{p}\right)^{1 /(p-1)} d \tau \geq \int_{\frac{1}{2}}^{1}\left(1-\tau^{p}\right)^{1 /(p-1)} d \tau=C
$$

where $C$ is an appropriate constant depending only on $p$. For $\delta \leq \frac{1}{2}$, an application of the mean value theorem yields

$$
1-\tau^{p} \geq p(1-\delta)^{p-1}(1-\tau), \quad \forall \tau \in[1-\delta, 1] .
$$

Hence,

$$
\int_{1-\delta}^{1}\left(1-\tau^{p}\right)^{1 /(p-1)} \geq p^{1 /(p-1)}(1-\delta) \int_{1-\delta}^{1}(1-\tau)^{1 /(p-1)} d \tau=D(1-\delta) \delta^{p /(p-1)},
$$

where $D$ is an appropriate constant that depends only on $p$. Thus (3.12) yields

$$
\bar{T}_{\delta} T_{2}^{(n-1) /(p-n)} \leq \begin{cases}\bar{C} ; & \text { if } \frac{1}{2} \leq \delta \leq 1 \\ \bar{C} \delta^{(p-1) / p} ; & \text { if } 0<\delta \leq \frac{1}{2}\end{cases}
$$

where $\bar{C}$ is a constant that depends only on $n$ and $p$. Let $r_{\delta}$ in $\left[h_{m+1}, z_{m+1}\right]$ be such that $\phi\left(r_{\delta}\right)=(1-\delta) M$. Then,

$$
r_{\delta}-h_{m+1}=\left(T_{\delta}\right)^{(p-1) /(p-n)}-\left(T_{2}\right)^{(p-1) /(p-n)} \leq \frac{p-1}{n-p} \bar{T}_{\delta} T_{\delta}^{(n-1) /(p-n)} .
$$

Therefore, from (3.13) it follows that by choosing $T_{2}$ small enough, i.e. $h_{m+1}$ large enough, we may make $\bar{T}_{\delta} \leq \frac{1}{2} T_{2}$. Since $T_{\delta}=T_{2}-\bar{T}_{\delta}$, we have $T_{\delta} \geq \frac{1}{2} T_{2}$. Thus,

$$
r_{\delta}-h_{m+1} \leq \hat{C} \bar{T}_{\delta} T_{2}^{(n-1) /(p-n)},
$$


where $\hat{C}$ is a new constant. We have then shown that for all $m=0,1,2, \ldots$,

$$
r_{\delta}-h_{m+1} \leq \begin{cases}C ; & \frac{1}{2} \leq \delta \leq 1 \\ C \delta^{(p-1) / p} ; & 0<\delta \leq \frac{1}{2}\end{cases}
$$

Here $C$ is a constant that depends only on $n$ and $p$.

The analyses in the remaining cases are very much similar to Case 1 . Hence we only present details at places where the analyses differ.

Case 2. Let $p>n$, then (3.9) holds in the interval $\left[T_{1}, T_{2}\right]$ where now $T_{1}=\left(h_{m+1}\right)^{(p-n) /(p-1)}$ and $T_{2}=\left(Z_{m+1}\right)^{(p-n) /(p-1)}$. In this case, $\dot{w}\left(T_{1}\right)=$ $\dot{\phi}\left(h_{m+1}\right)=0, \operatorname{sign}(\dot{w})=\operatorname{sign}(\dot{\phi})$, and $w$ is decreasing. Upon integrating twice, (3.9) yields

$$
w(t)=w\left(T_{1}\right)-\frac{1}{A} \int_{T_{1}}^{t}\left\{\int_{T_{1}}^{x} s^{(n-1) p /(p-n)}|w(s)|^{p-2} w(s) d s\right\}^{1 /(p-1)} d x .
$$

With $\delta$ and $T_{\delta}$ as before, and noting that $w$ is concave in $\left[T_{1}, T_{\delta}\right]$ and setting $\bar{T}_{\delta}=T_{\delta}-T_{1},(3.14)$ gives

$$
\int_{T_{1}}^{T_{\delta}}\left[\int_{T_{1}}^{x} s^{(n-1) p /(p-n)}\left\{1+\frac{T_{1}-s}{\bar{T}_{\delta}} \delta\right\}^{p-1} d s\right]^{1 /(p-1)} d x \leq A \delta .
$$

It follows then

$$
\bar{T}_{\delta} T_{1}^{(n-1) /(p-n)} \leq \begin{cases}C ; & \text { if } \frac{1}{2} \leq \delta \leq 1 \\ C \delta^{(p-1) / p} ; & \text { if } 0<\delta \leq \frac{1}{2}\end{cases}
$$

where again $C$ is a constant that depends on $n$ and $p$. Defining $r_{\delta}$ as before,

$$
r_{\delta}-h_{m+1}=\left(T_{\delta}\right)^{(p-1) /(p-n)}-\left(T_{1}\right)^{(p-1) /(p-n)} \leq \frac{p-1}{p-n} \bar{T}_{\delta}\left(T_{\delta}\right)^{(n-1) /(p-n)} .
$$

Since $T_{\delta}=T_{1}+\bar{T}_{\delta}$, by choosing $T_{1}$ sufficiently large and using $\left(3.14^{\prime}\right), T_{\delta}$ can be majorized by say $3 T_{1} / 2$. Thus, it follows, for all $m=0,1,2, \ldots$,

$$
r_{\delta}-h_{m+1} \leq \begin{cases}C ; & \frac{1}{2} \leq \delta \leq 1 \\ C \delta^{(p-1) / p} ; & 0<\delta \leq \frac{1}{2}\end{cases}
$$

Case 3. Take $p=n$. Then (3.10) holds in $\left[T_{1}, T_{2}\right]$ where $T_{1}=\ln \left(h_{m+1}\right)$ and $T_{2}=\ln \left(z_{m+1}\right)$. We note that in this case $\dot{w}\left(T_{1}\right)=\phi\left(h_{m+1}\right)=0, \operatorname{sign}(\cdot w)=$ $\operatorname{sign}(\cdot \phi)$, and $w$ is decreasing. Thus

$$
w(t)=w\left(T_{1}\right)-\int_{T_{1}}^{t}\left\{\int_{T_{1}}^{x} e^{n s}|w(s)|^{n-2} w(s) d s\right\}^{1 /(n-1)} d x .
$$


With $\delta, T_{\delta}, \bar{T}_{\delta}$ and $r_{\delta}$ as before, we can show that

$$
\bar{T}_{\delta} e^{T_{1}} \leq \begin{cases}C ; & \text { if } \frac{1}{2} \leq \delta \leq 1 \\ C \delta^{(n-1) / n} ; & \text { if } 0<\delta \leq \frac{1}{2}\end{cases}
$$

where $C$ depends only on $n$. Thus, it follows, for all $m=0,1,2, \ldots$,

$$
r_{\delta}-h_{m+1} \leq \begin{cases}C ; & \frac{1}{2} \leq \delta \leq 1 \\ C \delta^{(n-1) / n} ; & 0<\delta \leq \frac{1}{2}\end{cases}
$$

We may sum up the conclusions as follows. For $1<p<\infty$, and for all $m=0,1,2, \ldots$,

$$
r_{\delta}-h_{m+1} \leq \begin{cases}C ; & \text { if } \frac{1}{2} \leq \delta \leq 1 \\ C \delta^{(p-1) / p} ; & \text { if } 0<\delta \leq \frac{1}{2}\end{cases}
$$

Here $C$ is an appropriate constant that depends only on $n$ and $p$.

Hence the distance between successive zeros is bounded uniformly from above. $\square$

The next lemma shows that $\left|\phi\left(h_{m}\right)\right|$ decreases as $m$ increases. It also sets up an inequality that will be used to prove that $\left|\phi\left(h_{m}\right)\right|$ actually decays to zero and $\left|z_{m+1}-z_{m}\right|$ approaches asymptotically a number $T(p)$ that depends only on $p$.

Lemma 3.4. The values $\left|\phi\left(h_{m}\right)\right|$ are decreasing.

Proof. For a fixed $m$, consider the interval $\left[h_{m}, h_{m+1}\right]$. Without any loss of generality, we may assume that $\phi\left(h_{m}\right)>0$ and $\phi\left(h_{m+1}\right)<0$. We note the following

(i) $\dot{\phi} \leq 0$ in $\left[h_{m}, h_{m+1}\right]$,

(ii) $\dot{\phi}\left(h_{m}\right)=\dot{\phi}\left(h_{m+1}\right)=0$, and

(iii) $\phi\left(z_{m}\right)=0$.

Multiplying the differential equation in (3.1) by $\dot{\phi}$ and simplifying, it follows

$$
(p-1)|\dot{\phi}|^{p-1} \frac{d}{d r}|\dot{\phi}|+\frac{n-1}{r}|\dot{\phi}|^{p}+|\phi|^{p-1} \frac{d}{d r}|\phi|=0,
$$

in $\left(h_{m}, h_{m+1}\right)$. Integrating the above, from $h_{m}$ to $h_{m+1}$, we obtain

$$
\left|\phi\left(h_{m}\right)\right|^{p}=\left|\phi\left(h_{m+1}\right)\right|^{p}+p(n-1) \int_{h_{m}}^{h_{m+1}} \frac{|\dot{\phi}(r)|^{p}}{r} d r .
$$

This shows that $\left|\phi\left(h_{m}\right)\right|$ is decreasing. By iterating the above relation, we find

$$
\left|\phi\left(h_{m}\right)\right|^{p}=|\phi(0)|^{p}-p(n-1) \int_{0}^{h_{m}} \frac{|\dot{\phi}(r)|^{p}}{r} d r,
$$


and hence

$$
\int_{0}^{\infty} \frac{|\dot{\phi}(r)|^{p}}{r} d r \leq \frac{|\phi(0)|^{p}}{p(n-1)}
$$

Proposition 3.1. For $x>0$ large, $1<p<\infty$ and $n \geq 2$, consider the integral

$$
I(x)=\int_{x}^{x+1} t^{1 /(p-1)}\left\{1-\left(\frac{x}{t}\right)^{n}\right\}^{p /(p-1)} d t .
$$

Then there are constants $C$ and $\bar{C}$ depending on $n$ and $p$ such that

$$
\frac{C}{x} \leq I(x) \leq \frac{\bar{C}}{x}
$$

Proof. For any $x>0$,

$$
I(x) \leq(x+1)^{1 /(p-1)}\left\{1-\left(\frac{x}{x+1}\right)^{n}\right\}^{p /(p-1)} .
$$

Applying the mean value theorem, we obtain

$$
I(x) \leq \frac{n^{p /(p-1)}}{x} .
$$

To obtain a lower bound for $I(x)$, we notice that

$$
I(x) \geq x^{1 /(p-1)} \int_{x}^{x+1}\left\{1-\left(\frac{x}{t}\right)^{n}\right\}^{p /(p-1)} d t .
$$

Since $(x / t)^{n} \leq(x / t) \leq 1$ and $x \leq t \leq x+1$, the above yields

$$
I(x) \geq\left\{\frac{x}{(x+1)^{p}}\right\}^{1 /(p-1)} \int_{x}^{x+1}(t-x)^{p /(p-1)} d t .
$$

Simplifying,

$$
I(x) \geq \frac{C}{x}
$$

This finishes the proof. $\square$

Proof of part (ii) of Theorem 2. We prove that $\left|\phi\left(h_{m}\right)\right| \rightarrow 0$ as $m \rightarrow \infty$, thereby proving that $\lim _{r \rightarrow \infty}|\phi(r)|=0$. In (3.17), take $\phi(0)=1$. We proceed by contradiction. Suppose there is an $\eta>0$ such that $\left|\phi\left(h_{m}\right)\right| \geq 2 \eta$, for all $m=0,1,2, \ldots$ Then $|\phi(r)| \geq\left|\frac{1}{2} \phi\left(h_{m}\right)\right| \geq \eta$ in $\left[h_{m}, b_{m / 2}\right]$, where $b_{m / 2}$ is as 
defined in Lemma 3.2. Furthermore, it follows from (3.6) that there is a $\delta>0$ such that for every $m, b_{m / 2}-h_{m} \geq \delta$. Recalling that $\phi\left(h_{m}\right)=\phi\left(z_{m}\right)=0$, an integration of $(3.1)$ over $\left[h_{m}, r\right]$ yields

$$
|\dot{\phi}(r)|^{p-1} \dot{\phi}(r)=-\frac{1}{r^{n-1}} \int_{h_{m}}^{r} t^{n-1}|\phi(t)|^{p-2} \phi(t) d t .
$$

It follows, regardless of the sign of $\phi$ in $\left[h_{m}, z_{m}\right]$, that

$$
|\dot{\phi}(r)|^{p-1} \geq \eta^{p-1}\left(\frac{r^{n}-h_{m}^{n}}{n r^{n-1}}\right) \quad \text { in }\left[h_{m}, b_{m / 2}\right] .
$$

Thus,

$$
|\dot{\phi}(r)|^{p} \geq C r^{p /(p-1)}\left\{1-\left(\frac{h_{m}}{r}\right)^{n}\right\}^{p /(p-1)} \quad \text { in }\left[h_{m}, b_{m / 2}\right],
$$

where $C=\eta^{p} / \eta^{p / p-1}$, for all $m=0,1,2, \ldots$ Now,

$$
\int_{0}^{\infty} \frac{|\dot{\phi}(t)|^{p}}{t} d t \geq \sum_{m=0}^{\infty} \int_{h_{m}}^{h_{m}+\delta} \frac{|\dot{\phi}(t)|^{p}}{t} d t
$$

Using (3.18),

$$
\int_{0}^{\infty} \frac{|\dot{\phi}(t)|^{p}}{t} d t \geq \sum_{m=0}^{\infty} C \int_{h_{m}}^{h_{m}+\delta} t^{1 /(p-1)}\left\{1-\left(\frac{h_{m}}{t}\right)^{n}\right\}^{p /(p-1)} d t
$$

The integral on the right side may be estimated using Proposition 3.1, and hence for large values of $m$, say $m \geq m_{0}$ for some $m_{0}$ large,

$$
\int_{0}^{\infty} \frac{|\dot{\phi}(t)|^{p}}{t} d t \geq \sum_{m_{0}}^{\infty} \frac{A(n, p, \delta, \eta)}{h_{m}} .
$$

From Lemma 3.3, $h_{m} \leq m L$ for some $L>0$. Thus the integral on the left hand side is divergent, contradicting (3.17). Hence $\left|\phi\left(h_{m}\right)\right| \rightarrow 0$ as $m \rightarrow \infty$.

We now prove part (iii) of Theorem 2 which describes the asymptotic behavior of the zeros.

Proof of part (iii) of Theorem 2. We show that $\lim _{m \rightarrow \infty} z_{m+1}-z_{m}=T(p)$, where $T(p)$ is an appropriate constant that depends only on $p$. Fix $m$, without any loss of generality take $\phi\left(h_{m+1}\right)=1$, thereby choosing $\phi>0$ in $\left[z_{m}, z_{m+1}\right]$. In (3.7), majorizing $\phi$ by 1 and applying Lemma 3.3, we obtain that $|\phi(r)| \leq M$, $z_{m} \leq r \leq z_{m+1}$. Here $M$ depends on $n$ and $p$. We now divide the proof into two 
parts. In part (a) we prove that $h_{m+1}-z_{m}$ has an asymptotic limit, and in part (b) we show that $z_{m+1}-h_{m+1}$ has the same limit.

(a) Consider the interval $\left[z_{m}, h_{m+1}\right]$. We have that $\phi\left(z_{m}\right)=\dot{\phi}\left(h_{m+1}\right)=0$, $\dot{\phi}>0$ and thus $\phi$ is increasing. Integrating (3.16) from $r$ to $h_{m+1}$, yields

$$
(p-1)|\dot{\phi}(r)|^{p}+|\phi(r)|^{p}=1+C \int_{r}^{h_{m+1}} \frac{|\dot{\phi}(t)|^{p}}{t} d t,
$$

where $C=p(n-1)$. Using that $|\dot{\phi}| \leq M$, we obtain with a new constant $\bar{C}$,

$$
1<(p-1)|\dot{\phi}(r)|^{p}+|\phi(r)|^{p} \leq 1+\bar{C} \ln \frac{h_{m+1}}{z_{m}} \leq 1+\varepsilon(m) .
$$

Since $h_{m+1}-z_{m} \leq L$, for some $L$ independnt of $m$, it follows that $\varepsilon(m) \rightarrow 0$ as $m \rightarrow \infty$. Integrating the first inequality in (3.19) from $z_{m}$ to $h_{m+1}$, we find that

$$
(p-1)^{1 / p} p \int_{0}^{1} \frac{d \phi}{\left(1-\phi^{p}\right)^{1 / p}}>\int_{z_{m}}^{h_{m+1}} d t=h_{m+1}-z_{m} .
$$

Thus,

$$
h_{m+1}-z_{m} \leq(p-1)^{1 / p} P(p),
$$

where $P(p)=\int_{0}^{1}\left(1-t^{p}\right)^{-1 / p} d t$. Let $\epsilon>0$, and $m$ be sufficiently large so that $\varepsilon(m)<\varepsilon$. By integrating the second inequality in (3.19), again from $z_{m}$ to $h_{m+1}$, we get

$$
(p-1)^{1 / p} \int_{0}^{1} \frac{d \phi}{\left(1+\varepsilon-\phi^{p}\right)^{1 / p}} \leq \int_{z_{m}}^{h_{m+1}} d t=h_{m+1}-z_{m} .
$$

We estimate the integral on the left side of the inequality. It is clear that

$$
\begin{aligned}
\int_{0}^{1} \frac{d t}{\left(1+\epsilon-t^{p}\right)^{1 / p}} & =\int_{0}^{(1 /(1+\epsilon))^{1 / p}} \frac{d s}{\left(1-s^{p}\right)^{1 / p}} \\
& =\int_{0}^{1} \frac{d s}{\left(1-s^{p}\right)^{1 / p}}-\int_{(1 /(1+\epsilon))^{1 / p}}^{1} \frac{d s}{\left(1-s^{p}\right)^{1 / p}} \\
& \geq P(p)-C\left(\frac{\varepsilon}{1+\varepsilon}\right)^{(p-1) / p}
\end{aligned}
$$

where $C$ is an appropriate constant that depends only on $p$. The estimate on the second integral has been gotten by using the substitution $v=s^{p}$, and majorizing $v$ by 1 . From (3.20) and (3.21), we get

$$
(p-1)^{1 / p} P(p)-C\left(\frac{\varepsilon}{1+\varepsilon}\right)^{(p-1) / p} \leq h_{m+1}-z_{m} \leq(p-1)^{1 / p} P(p) .
$$


(b) Consider now the interval $\left[h_{m+1}, z_{m+1}\right]$. In this case $\phi\left(z_{m+1}\right)=0$, $\dot{\phi}\left(h_{m+1}\right)=0, \dot{\phi}<0$ and thus $\phi$ is decreasing. Integrating (3.16) from $h_{m+1}$ to $r$, we find

$$
(p-1)|\dot{\phi}(r)|^{p}+|\phi(r)|^{p}=1-C \int_{h_{m+1}}^{r} \frac{|\dot{\phi}(t)|^{p}}{t} d t,
$$

where once again $C=p(n-1)$. Using that $|\dot{\phi}| \leq M$, it follows that

$$
1-\varepsilon(m) \leq(p-1)|\dot{\phi}(r)|^{p}+|\phi(r)|^{p}<1,
$$

where $\varepsilon(m)=\bar{C} \ell n z_{m+1} / h_{m+1}$ and $\bar{C}$ is an appropriate constant. As before, $\varepsilon(m) \rightarrow 0$ as $m \rightarrow \infty$. Integrating the second inequality in (3.22) from $h_{m+1}$ to $z_{m+1}$, we obtain

$$
z_{m+1}-h_{m+1}>(p-1)^{1 / p} P(p) .
$$

Let $\eta>0$ be such that $1-(1-\eta)^{1 / p}<\frac{1}{4}$. Choose $m$ so large that $\varepsilon(m)<\eta$. Define $\bar{r}_{\eta}$ in $\left[h_{m+1}, z_{m+1}\right]$ to be the value of $r$ for which $\phi\left(\bar{r}_{\eta}\right)=(1-\eta)^{1 / p}$. In the first inequality in (3.22), replace $\varepsilon(m)$ by $\eta$ and integrate from $\bar{r}_{\eta}$ to $z_{m+1}$ to obtain

Therefore,

$$
(p-1)^{1 / p} \int_{0}^{(1-\eta)^{1 / p}} \frac{d \phi}{\left(1-\eta-\phi^{p}\right)^{1 / p}} \geq z_{m+1}-\bar{r}_{\eta} .
$$

From (3.15), with $\delta=1-(1-\eta)^{1 / p}$,

$$
z_{m+1}-\bar{r}_{\eta} \leq(p-1)^{1 / p} P(p) .
$$

$$
\bar{r}_{\eta}-h_{m+1} \leq C \eta^{(p-1) / p},
$$

where $C$ is an appropriate constant. It follows that

$$
z_{m+1}-h_{m+1} \leq(p-1)^{1 / p} P(p)+C \eta^{(p-1) / p} .
$$

From (3.23) and the foregoing inequality,

$$
(p-1)^{1 / p} P(p)<z_{m+1}-h_{m+1} \leq(p-1)^{1 / p} P(p)+C \eta^{(p-1) / p} .
$$

Combining the results of part (a) and part (b) we see that

Thus,

$$
\lim _{m \rightarrow \infty} z_{m+1}-z_{m}=2(p-1)^{1 / p} P(p) \text {. }
$$

$$
T(p)=2(p-1)^{1 / p} P(p) \text {. }
$$

We now prove that $\phi$ is unique. By Corollary 2.3 the function $\phi$ is the first eigenfunction, with $\lambda_{1}=1$, on the ball of radius $z_{0}$. By Corollary 2.2, $z_{0}$ is unique. By Theorem $1, \phi$ is unique on $\left[0, z_{0}\right]$. Now suppose that for some $m \geq 0$, the zeros $z_{0}, z_{1}, \ldots, z_{m}$ and $\phi$ on $\left[0, z_{m}\right]$ are unique. It is clear that $\phi$ is the first eigenfunction on the annulus formed by $z_{m}$ and $z_{m+1}$, with $\lambda_{1}=1$. Again uniqueness of $z_{m+1}$ and $\phi$ on $\left[z_{m}, z_{m+1}\right]$ follow from Corollary 2.2 and Theorem 1 .

Acknowledgement. The author thanks his thesis advisor Professor Allen Weitsman for his guidance and encouragement. 


\section{References}

[1] Anane, A.: Simplicité et isolation de la première valeur propre du $p$-Laplacian avec poids. - C. R. Acad. Sci. Paris 305, Sér. I Math., 1987, 725-728.

[2] Bhattacharya, T.: Radial symmetry of the first eigenfunction of the $p$-Laplacian on the ball. - Proc. Amer. Math. Soc. (to appear).

[3] DiBenedetto, E.: $C^{1+d}$ local regularity of weak solutions of degenerate elliptic equations. - Nonlinear Anal. Vol. 7, No. 8, 827-850, 1983.

[4] García Azorero, J.P., and I. Peral Alonso: Existence and non-uniqueness for the $p$-Laplacian: Non-linear eigenvalues. - Comm. Partial Differential Equations 12, 1987, $1389-1430$.

[5] García Azorero, J.P., and J. Peral Alonso: Asymptotic behaviour of the eigenvalues of the $p$-Laplacian. - Preprint.

[6] Gidas, B., Wei-Ming Ni, and L. Nirenberg: Symmetry and related properties via the maximum principle. - Comm. Math. Phys. 68, 1979, 209-243.

[7] Gilbarg, D., and N.S. Trudinger: Elliptic partial differential equations of the second order, 2nd edition. - Springer-Verlag, Berlin-Heidelberg-New York, 1983.

[8] Pólya, G., and G. Szegö: Isoperimetric inequalities in mathematical physics. - Princeton University Press, Princeton, 1951.

[9] SAKAGUCHI, S.: Concavity properties of solutions to some degenerate quasilinear elliptic Dirichlet problems. - Ann. Scuola Norm. Sup. Pisa Cl. Sci. (4) (to appear).

[10] Serrin, J.: Local behaviour of solutions of quasi-linear equations. - Acta Math. 111, 1964, 247-302.

[11] Thelin, F.D.: Sur l'espace propre associé à la premier valeur propre du pseudo-Laplacien. - C. R. Acad. Sci. Paris Sér. I Math. 8, 1986, pages.

[12] Tolksdorf, P.: Regularity for a more general class of quasi-linear elliptic equations. - J. Differential Equations 51, 1984, 126-150.

[13] Tolksdorf, P.: On the Dirichlet problem for quasi-linear equations in domains with conical boundary points. - Comm. Partial Differential Equations 8, 1983, 773-817.

[14] Selby, Samuel M.: Standard mathematical tables. - CRC Press, Inc., Cleveland, Ohio, 1973.

[15] Veron, L., and M. Guedda: Quasilinear elliptic equations involving critical Sobolev exponents. - Nonlinear Anal. (to appear).

Northwestern University

Department of Mathematics

Lunt Hall

Evanston, IL 60208-2730

U.S.A. 